\title{
REGULAR AND MACH REFLECTION OF SHOCK WAVES ${ }^{1}$
}

\author{
Hans Hornung \\ Institute for Experimental Fluid Mechanics, DFVLR, Bunsenstraße 10, \\ D-3400 Göttingen, Federal Republic of Germany
}

\section{INTRODUCTION}

The properties that distinguish a shock wave from other waves are that its thickness is negligible compared with other characteristic lengths, and that the state of the medium is changed irreversibly by the passage of the wave. Shocks are therefore a highly nonlinear phenomenon, and parameter changes may be expected to lead to numerous bifurcations.

The multitude of possibilities is compounded when more than one shock occurs, such as in the interaction of a shock with a solid surface or symmetry plane (i.e. in shock reflection). The subject of shock reflection is so complicated that it is necessary to introduce it at some length. In the interest of conciseness, this is done according to a logical rather than a historical sequence, with explicit reference to the important authors omitted in the text unless their work is relatively recent.

Salient points in the early development of the subject should be mentioned here, however. These may be found in the experiments of Mach (1878), the theoretical work of von Neumann (1943), the experimental and theoretical work of the group around Bleakney at Princeton (e.g. Bleakney \& Taub 1949, Smith 1945), the experiments of Kawamura \& Saito (1956), Smith (1959), and Bryson \& Gross (1961), and the theoretical work of Lighthill (1949) and Jones et al. (1951). A review by Pack (1964) gives more detailed references. Of the work since 1960, that of the group around Glass at Toronto (e.g. Law \& Glass 1971, Ben-Dor \& Glass 1979, 1980) and around Henderson at Sydney (e.g. Henderson \& Lozzi 1975, 1979,

${ }^{1}$ Dedicated to the memory of Ernst Becker. 


\section{HORNUNG}

Henderson \& Gray 1981) stand out. A review by Griffith (1981) gives more detailed references.

In this review the discussion is restricted to plane flow. This is because relatively little work has been done on three-dimensional situations, and because a compromise had to be made between depth and breadth of the field covered.

\section{Material Properties}

Shocks may occur in many different kinds of materials. However, in the main part of this review the discussion is restricted to situations in which the material may be described to a good approximation by the model of a compressible, heat-conducting Newtonian continuum. The additional assumption that a material element is subjected only to such slow changes that (to a good approximation) it is able to maintain (local) thermodynamic equilibrium everywhere leads to a simple expression for the thickness of shocks in terms of the bulk and shear viscosities, thermal conductivity, density, and speed of sound of the material. If these properties are replaced by corresponding values for a real gas of discrete molecules, then the thickness of sufficiently strong shocks amounts only to a few mean free paths. Thus, to be consistent with our assumption that the material behaves as a continuum, we have to neglect the shock thickness and treat the shock as a surface of discontinuity.

In a continuum framework, local thermodynamic equilibrium is therefore not maintained within this thin shock. In the main part of this review it is assumed that thermodynamic equilibrium is maintained everywhere else, and that (to a good approximation) the temperature and equilibrium states are defined by the equation of state of a thermally and calorically perfect gas. For our purposes the material is therefore specified by the density $\rho$, shear viscosity $\mu$, thermal conductivity $k$, specific heat $c_{\mathrm{p}}$, and specific gas constant $R$. In this framework, a typical situation whose characteristic length, flow velocity, and wall and reservoir temperatures are $d, q, T_{\mathrm{w}}$, and $T_{0}$, respectively, leads to a dependence of any dimensionless quantity $Q$ on five parameters:

$$
Q=Q\left[\rho q d / \mu, \rho q^{2} /(\gamma p), \gamma, \mu c_{\mathrm{p}} / k, T_{\mathrm{w}} / T_{0}\right],
$$

or

$$
Q=Q\left(\operatorname{Re}, M^{2}, \gamma, \operatorname{Pr}, T_{w} / T_{0}\right),
$$

where

$$
\gamma=c_{\mathrm{p}} /\left(c_{\mathrm{p}}-R\right) \text {. }
$$

The further restriction to a situation in which shear rates and temperature gradients are so small that shear stresses and heat flux may be neglected 
defines such an important limiting case that a large part of this review is devoted to it. It is referred to somewhat loosely as "inviscid" flow. Equation (1) degenerates for inviscid flow to

$$
Q=Q(M, \gamma)
$$

Other material properties, such as vibrational or chemical relaxation, are of considerable importance in the reflection of strong shock waves. They, like viscosity and heat conduction, introduce new length scales into the problem, albeit in a different way. Relaxation effects are discussed only briefly at the end of this review.

\section{The Shock-Jump Relations}

In steady flow through an oblique shock wave (see Figure 1), the conservation equations may be used to relate the state downstream of the shock (subscript 2) to the state upstream (subscript 1):

$$
\begin{gathered}
\rho_{2} / \rho_{1}=(\gamma+1) M_{1}^{2} \sin ^{2} \alpha /\left[2+(\gamma-1) M_{1}^{2} \sin ^{2} \alpha\right], \\
p_{2} / p_{1}=\left[2 \gamma M_{1}^{2} \sin ^{2} \alpha-(\gamma-1)\right] /(\gamma+1), \\
M_{2}^{2} \sin ^{2}(\alpha-\theta)=\left[\gamma+1+(\gamma-1)\left(M_{1}^{2} \sin ^{2} \alpha-1\right)\right] \\
\quad \quad\left[\gamma+1+2 \gamma\left(M_{1}^{2} \sin ^{2} \alpha-1\right)\right], \\
\tan \theta=\tan \alpha\left[M_{1}^{2} \cos ^{2} \alpha-\cot ^{2} \alpha\right] /\left[1+\frac{1}{2} M_{1}^{2}(\gamma+\cos 2 \alpha)\right] .
\end{gathered}
$$

For fixed $\gamma$ and $M_{1}$, Figure 2 shows the shock angle $\alpha$ as a function of the deflection angle $\theta$. As $\alpha$ is increased from $\arcsin \left(M_{1}^{-1}\right), M_{2}$ decreases from $M_{1}$ to a value of 1 (sonic point) just before $\theta$ reaches a maximum value (maximum-deflection point). Further increase of $\alpha$ causes $M_{2}$ and $\theta$ to decrease until $\theta=0$ at $\alpha=\pi / 2$ (normal shock).

Again for fixed $\gamma$ and $M_{1}$, a similar curve may be drawn for $p_{2}(\theta) / p_{1}$ by eliminating $\alpha$ between Equations (4) and (6) (see Figure 3). Both curves are symmetrical about $\theta=0$, of course. Their nonlinearity is evident.
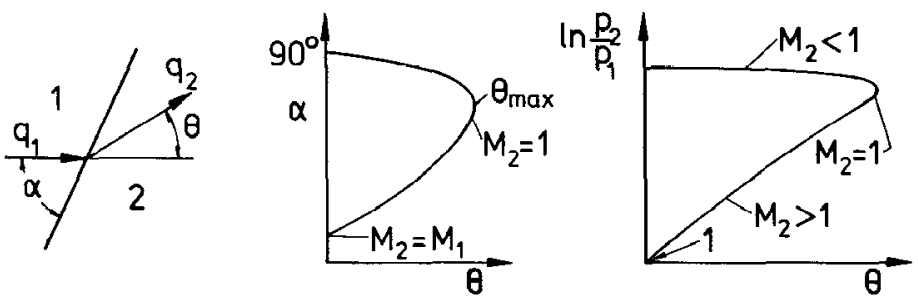

Figure 1 (left) Defining sketch.

Figure 2 (center) Shock locus in $(\theta, \alpha)$-plane. $M_{1}, \gamma$ constant.

Figure 3 (right) Shock locus in $(\theta, p)$-plane. $M_{1}, \gamma$ constant. 


\section{Reflection of a Plane Shock From a Plane Surface}

STEADY INVISCID FLOW Consider a plane shock (for example, one generated by a wedge in steady flow) being reflected off a wall or symmetry plane parallel to $q_{1}$ (see Figure 4). The incident shock I deflects the flow to an angle $\theta_{2}$, and a second shock is needed to deflect it back to $\theta_{3}=0$. This situation may be represented in the $(\theta, p)$-plane (see Figure 4): Condition 2 lies on the incident-shock locus I, below the point $M_{2}=1$. From this new condition we may draw a second shock locus with $M=M_{2}$ from $\left(\theta_{2}, p_{2}\right)$. Thus, the deflection is taken from 0 to $\theta_{2}$ by the incident shock $\mathrm{I}$, and from $\theta_{2}$ to 0 by the reflected shock $R$. This configuration is called regular reflection. The $(\theta, p)$-diagram in Figure 4 is a graphical representation of the mathematical problem of solving a double application of the shock-jump relations in a particular case.

If the angle $\alpha$ is increased, a value $\alpha=\alpha_{\mathrm{d}}\left(M_{1}, \gamma\right)$ is reached, beyond which the second shock locus does not reach the $p$-axis any more (see Figure 5). The deflection in region 3 is thus still positive (toward the wall). This necessitates a nonuniform buffer zone, across which the transition from $\theta>0$ to $\theta=0$ is accommodated. A near-normal shock $\mathbf{S}$ allows the tripleshock point $P$ to move away from the wall, with the result that a vortex sheet $V$ separates the gas processed by $S$ from that processed by I and $R$. The density and velocity, as well as the entropy, are discontinuous across $V$, but the pressure and streamline deflection must be continuous. This is the reason for the choice of the $(\theta, p)$-plane for the graphical representation of the solution of shock-reflection problems: Since $\theta_{3}=\theta_{4}$ and $p_{3}=p_{4}$, the points representing states 3 and 4 are congruent in $(\theta, p)$. Thus, the upper intersection of $I$ and $R$ in Figure 5 gives the triple-point condition, and the "Mach stem" S is represented by the part 4-5 of the curve I. Clearly S must be curved as a consequence. This reflection configuration is called Mach reflection, after its discoverer. (As is seen later, the transition to Mach reflection may occur at angles smaller than $\alpha_{\mathrm{d}}$.)
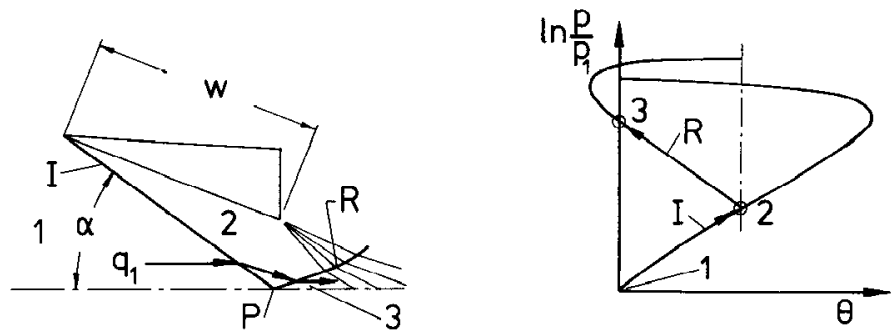

Figure 4 Regular reflection in steady flow. Physical (left) and $(\theta, p)$-plane (right). 

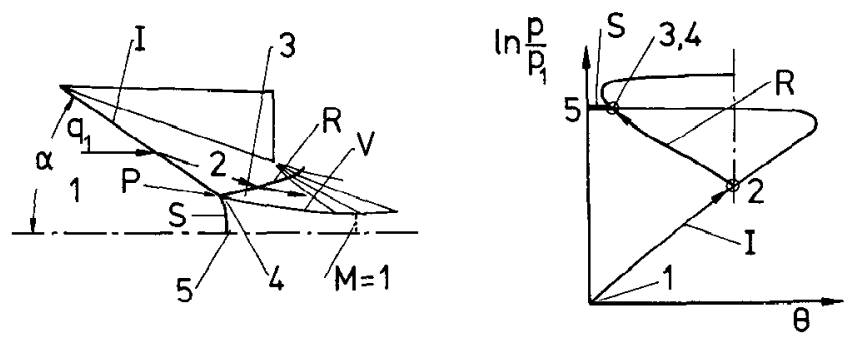

Figure 5 Mach reflection in steady flow. Physical (left) and $(\theta, p)$-plane (right).

PSEUDOSTEADY INVISCID FLOW Consider a plane shock I moving from right to left into a gas at rest in an inertial frame, with the speed of the shock being $q_{\mathrm{s}}$. Let it strike a symmetrical wedge, at rest in this inertial frame, whose leading edge $T$ is parallel and whose symmetry plane is normal to the plane of the shock (see Figure 6). If $\alpha$ is sufficiently small, regular reflection occurs. The intersection point $P$ of $I$ and the wall moves up the wedge surface at a constant speed

$$
q_{1}=q_{\mathrm{s}} / \sin \alpha,
$$

so that the distance $d$ increases linearly with time. In a frame of reference moving with $\mathrm{P}$ (which is therefore also an inertial frame), the flow in the immediate vicinity of $\mathrm{P}$ is steady, unless $M_{3}<1$. In the latter case, region 3 is unsteady. The $(\theta, p)$-plane may again be used to describe the reflection system (see Figure 7).

The transition from regular to Mach reflection again becomes necessary
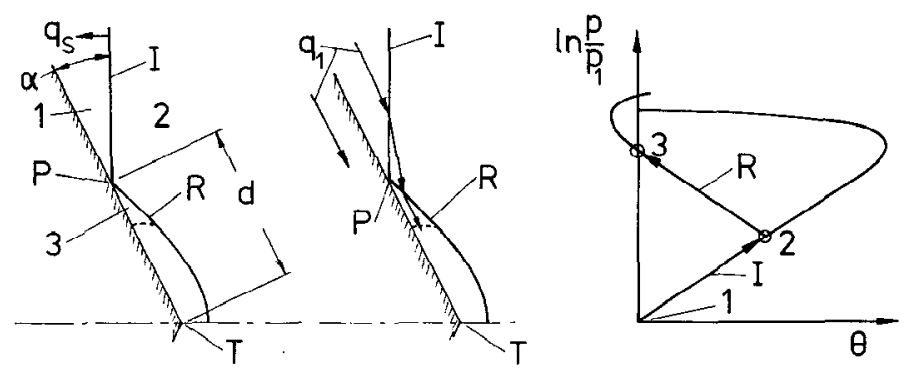

Figure 6 (left) Regular reflection in pseudosteady flow, viewed from an inertial frame fixed in $\mathbf{T}$.

Figure 7 (center and right) Regular reflection in pseudosteady flow, viewed from an inertial frame fixed in P. Physical (center) and ( $\theta, p)$-plane (right). 


\section{HORNUNG}

because of the failure of $R$ to reach the $p$-axis (see Figure 8). The triple point $P$ is now off the wall, so that the angle $\alpha$ between the oncoming flow (in the frame of reference of $P$ ) and the incident shock $I$ is no longer equal to the angle $\alpha+\chi$ between $I$ and the wall. Again, the gas processed by $I$ and $R$ is separated by a vortex sheet $\mathrm{V}$ from that processed by $\mathrm{S}$. In the frame of reference of $P$, the wall has a finite component of velocity normal to its own plane. The gas contained in the triangular region PBA is that which originally occupied the region PTA.

Flows of the kind depicted in Figures 7 and 8 are self-similar, inasmuch as they grow linearly with time. They are called pseudosteady. This is because in the absence of shear stresses and heat flux, the only characteristic length of the boundary conditions is $d$, which grows linearly with time (see Jones et al. 1951). Indeed, if the space coordinates are divided by the time $t$ elapsed from the instant that $I$ hits $T$, the equations of motion possess similar solutions. Thus, if the inviscid flow equations

$$
\begin{aligned}
& \frac{D \mathbf{q}}{D t}=-\operatorname{grad} p / \rho, \\
& \frac{D \rho}{D t}=-\rho \operatorname{div} \mathbf{q}
\end{aligned}
$$

are rewritten in the variables

$$
\left.\begin{array}{rl}
(\mathbf{X}, t) & =(\mathbf{x} / t, t) \\
\mathbf{U} & =\mathbf{q}-\mathbf{X}
\end{array}\right\},
$$

the explicit dependence on $t$ disappears for all $t>0$ :

$$
\left.\begin{array}{l}
\mathbf{U} \cdot \operatorname{Grad} \mathbf{U}+\mathbf{U}=-\mathrm{Grad} p / \rho \\
\mathbf{U} \cdot \operatorname{Grad} \rho+\rho(\operatorname{Div} \mathbf{U}+2)=0
\end{array}\right\}
$$
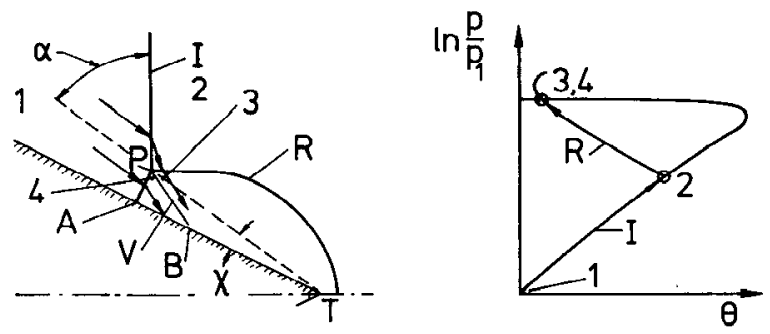

Figure 8 Mach reflection in pseudosteady flow, viewed from an inertial frame fixed in $P$. Physical (left) and $(\theta, p)$-plane (right). 


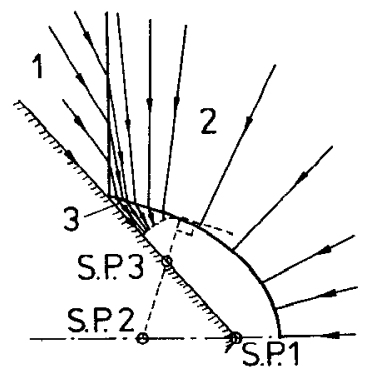

Figure 9 Regular reflection in pseudosteady similarity space, S.P. = sink points for the uniform-flow regions 1,2 , and 3 .

where Div and Grad denote vector operators in plane $\mathbf{X}$-space. Provided that the boundary and initial conditions are consistent with this scheme, self-similar solutions are possible. Figure 9 shows the "streamlines" in pseudosteady space, i.e. the integral curves of $\mathbf{U}$ in $\mathbf{X}$-space, for the case of regular reflection. Note that a region of uniform flow in physical space has straight integral curves that converge onto a single "sink" point in pseudosteady space. The whole of the flow pattern is independent of time in this space.

\section{INVISCID STEADY FLOW: CASE DISTINCTIONS}

In this section we discuss the differences in the reflection configurations that arise as consequences of the shock-jump relations, i.e. the influences of the parameters $M_{1}, \gamma$, and $\alpha$. The result of this discussion is a definition of the domains in the $\left(M_{1}, \alpha\right)$-plane (for fixed $\gamma$ ) to which certain forms of reflection are restricted.

\section{The Special Values of $\alpha\left(M_{1}, \gamma\right)$}

The condition in which the reflected-shock locus just reaches the p-axis is called the maximum-deflection condition of the reflection, and the shock angle at which it occurs is

$$
\alpha_{\mathrm{d}}=\alpha_{\mathrm{d}}\left(M_{1}, \gamma\right)
$$

The pressure at the point of tangency of the reflected-shock locus and the $p$ axis may lie below or above the normal-shock value, depending on $M_{1}$ and $\gamma$. Accordingly, one refers to "weak" or "strong" reflection, respectively (see Figure 10).

Just below this value of $\alpha$, a value

$$
\alpha_{\mathrm{s}}=\alpha_{\mathrm{s}}\left(M_{1}, \gamma\right)
$$

exists, at which the sonic point of the reflected-shock locus lies on the p-axis. 

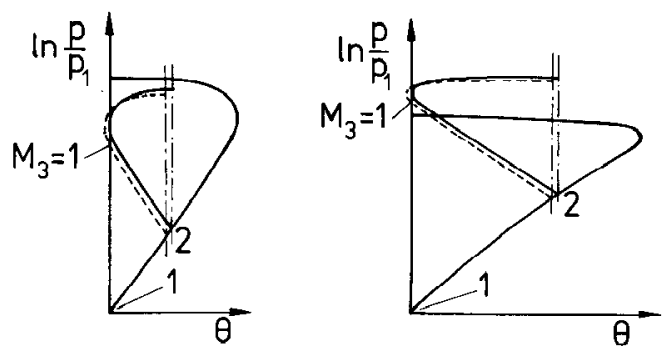

Figure 10 The conditions $\alpha=\alpha_{d}$ (full line) and $\alpha=\alpha_{\mathrm{s}}$ (dashed line) for weak (left) and strong reflection (right).

This condition is indicated by the dashed lines in Figure 10. It is referred to as the sonic condition for the reflection.

A third special value of $\alpha$ is defined by the condition that the reflectedshock locus intersects the $p$-axis at the normal-shock point (see Figure 11). This is named after von Neumann (1943), who first recognized its importance:

$$
\alpha_{\mathrm{N}}=\alpha_{\mathrm{N}}\left(M_{1}, \gamma\right)
$$

For values of $\gamma$ that are realistic for gases, there exists a single configuration for which

$$
\alpha_{\mathrm{N}}\left(M_{1}, \gamma\right)=\alpha_{\mathrm{d}}\left(M_{1}, \gamma\right)
$$

This occurs at a particular value of $M_{1}(\gamma)$ (see Figure 11). There also exist two configurations for which

$$
\alpha_{\mathrm{N}}\left(M_{1}, \gamma\right)=\alpha_{\mathrm{s}}\left(M_{1}, \gamma\right) \text {. }
$$

The less important one of these occurs in the weak-reflection range. For this, the sonic point on the reflected shock corresponds to a pressure that is
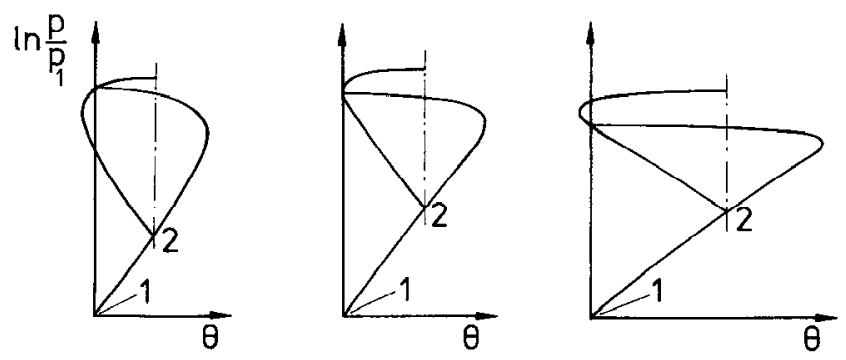

Figure 11 The condition $\alpha=\alpha_{\mathrm{N}}$. Weak (left) and strong reflection (right), $\alpha_{\mathrm{N}}=\alpha_{\mathrm{d}}$ (center). 

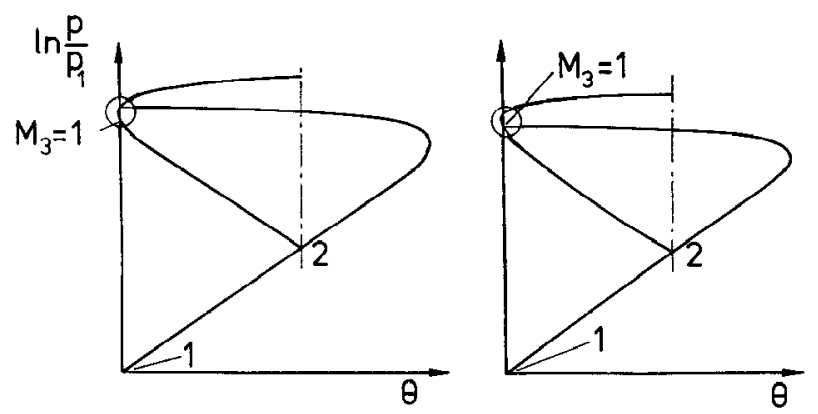

Figure 12 The two conditions at which $\alpha_{\mathrm{N}}=\alpha_{\mathrm{g}}$.

lower than that at the normal-shock point. The other occurs in the strongreflection range, and for this the pressure at the sonic point of the reflected shock coincides with the normal-shock pressure (see Figure 12). For a given $\gamma$, the values of $M_{1}$ for these two configurations lie on either side of that for $\alpha_{d}=\alpha_{N}$ and very close to it.

\section{The Mach-Number Ranges for $\gamma=1.4$}

For a fixed value of $\gamma$, coincidences of the special values of $\alpha$, such as Equations (15) and (16), as well as other conditions, define special values of the Mach number. In this section the Mach-number ranges defined by these special values are discussed for the case $\gamma=1.4$. No qualitative differences occur when $\gamma$ is changed to values relevant for other gases.

THE RANGE $1<M_{1}<1.25$ In this range there exists no value of $\alpha$ for which the reflected-shock locus $\mathrm{R}$ can intersect the incident-shock locus $\mathrm{I}$ other than at the point 2 (see Figure 13). Mach reflection is therefore not possible in this range. If $\alpha>\alpha_{d}$ (dashed curve for $R$ ), no reflected shock occurs at all, the incident shock is curved, and a subsonic region occurs behind it. The states
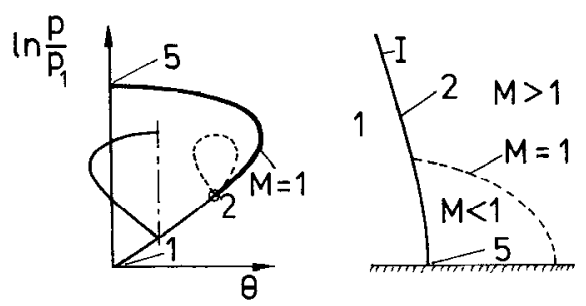

Figure 13 The range where no Mach reflection is possible.(right) Physical plane corresponding to dashed curve in $(\theta, p)$-plane (left). 
Figure 14 Reflected shock inclined forward, $1.25<M_{1}<1.48(\gamma=1.4)$.
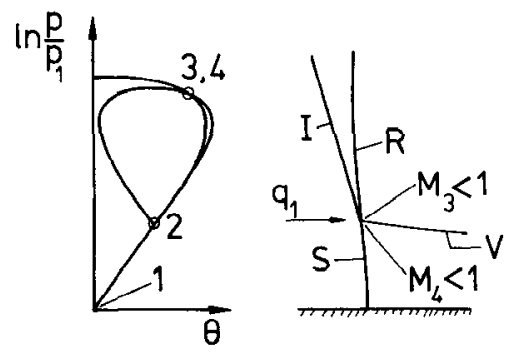

behind the curved shock are defined by the part of the shock locus that is drawn heavy in Figure 13.

THE RANGE $1.25<M_{1}<1.48$ In this range an intersection point between the I and $R$ locus can exist. However, it lies to the right of the symmetry line of the $\mathrm{R}$ locus (see Figure 14), i.e. at $\theta_{3}>\theta_{2}$. This means that the reflected shock is inclined forward at the triple point $P$.

THE RANGE $1.48<M_{1}<2.2$ For all $M_{1}>1.48$, the possibility of an inverted Mach reflection exists if $\alpha<\alpha_{\mathrm{N}}$. It is shown as a dashed line in the $(\theta, p)$ plane of Figure 15. This reflection mode always has the alternative solution of a regular reflection. It can therefore only be achieved by suppressing this alternative (e.g. by restricting the flow downstream, thus raising the back pressure to the value corresponding to that behind the inverted Mach reflection). This configuration is characterized by the fact that $\theta_{3}<0$, so that V slopes away from the wall and the Mach stem is convex toward the upstream direction (see Henderson \& Lozzi 1979).

For $\alpha>\alpha_{\mathrm{d}}$, a direct Mach reflection occurs, this being the only solution. This time we have $\theta_{3}<\theta_{2}$, and the reflected shock is inclined downstream

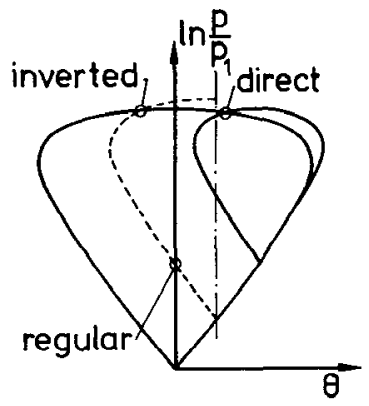

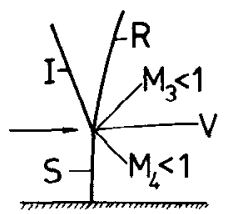

inverted

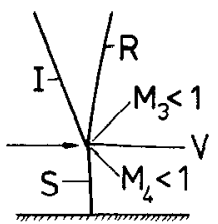

direct

Figure 15 Direct and inverted Mach reflection in the range $1.48<M_{1}<2.2(\gamma=1.4)$. 
when $\alpha$ is near $\alpha_{d}$, but as $\alpha$ increases further, the reflection reverts back to the configuration observed in the range $1.25<M_{1}<1.48$, with forwardinclined $\mathbf{R}$.

For $\alpha_{N}<\alpha<\alpha_{d}$, both regular and direct Mach reflection are possible, and since $M_{3}<1$, downstream conditions could enforce Mach reflection in this region.

THE RANGE $2.2<M_{1}<2.4$ At $M_{1}=2.2$, region 3 is sonic at $\alpha=\alpha_{\mathrm{N}}$. This is the upper one of the two points defined by Equation (16). Above $M_{1}=2.4 \mathrm{a}$ Mach reflection is characterized by the fact that $V$ separates the supersonic region 3 from the subsonic region 4 . This is the case illustrated in Figure 5. Again, both regular and direct Mach reflection are possible in the range $\alpha_{\mathrm{N}}<\alpha<\alpha_{\mathrm{d}}$ (see also Figures 10,11).

\section{The Reflection Regions in the $\left(M_{1}, \alpha\right)$-Plane}

The constraints on the various reflection modes may be delineated in a diagram of $\alpha$ versus $M_{1}$ (see Figure 16). This summarizes the discussion of the previous section. (Note that the domains of complex and double Mach reflection are omitted here. They are discussed in a later section.) It is well to remember that these boundaries apply to inviscid flow with $\gamma=1.4$. In addition, it became clear at a number of points that the $(\theta, p)$-plane, which deals with the immediate vicinity of the point $P$ only, cannot give any clues about which mode actually occurs when more than one reflection mode is possible. The downstream boundary condition needs to be invoked. We may therefore expect differences between steady and pseudosteady flow in such cases. This is because though the region around $\mathbf{P}$ is steady in both

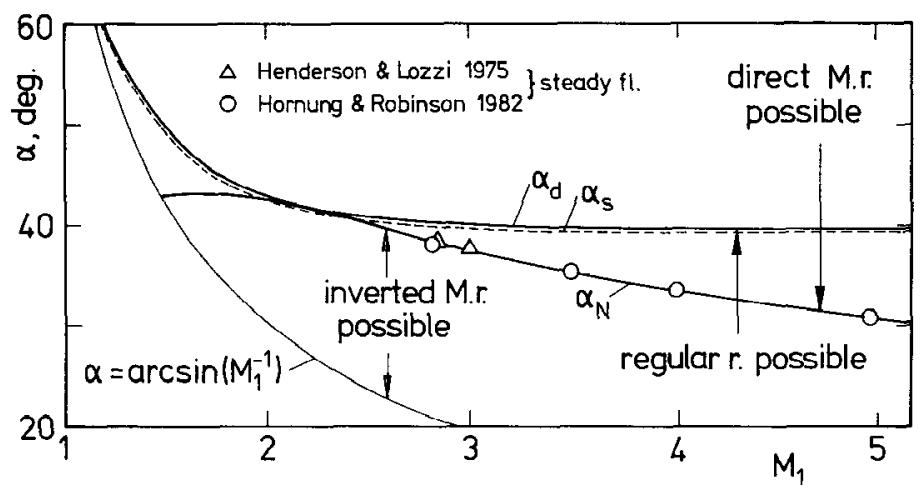

Figure 16 The special values of $\alpha$ as functions of $M_{1}$ for $\gamma=1.4$, and constraints on the reflection configurations. The points show observed failure of regular reflection in steady flow. 
cases if observed from P's reference frame, the downstream boundary conditions may not be influenced in pseudosteady flow (without breaking the pseudostationarity).

\section{THE INFORMATION CONDITION}

An important determining factor in regions of overlap where, on the basis of the shock-jump conditions, both regular and Mach reflection are possible is the fact that-unlike regular reflection-Mach reflection does display a characteristic length in the vicinity of $P$. For this to be possible, information about the characteristic length of the problem ( $w$ for steady, $d$ for unsteady flow) must reach the point $P$.

\section{Pseudosteady Flow}

If we consider the case of a regular reflection in pseudosteady flow, the point $P$ moves away from the wedge tip $T$ at a constant speed $q_{1}$. The information from the wedge tip travels at the local speed of sound relative to the fluid and chases $\mathbf{P}$ (see Figure 7). Clearly, this signal is only able to keep up with $P$ if $M_{3} \leq 1$, i.e. if $\alpha \geq \alpha_{s}$. Thus, information about $T$ does not reach $P$, and Mach reflection is not possible in pseudosteady flow unless $\alpha \geq \alpha_{\mathrm{s}}$. This simple argument all but eliminates the regions of overlap in the case of pseudosteady flow, because the difference $\alpha_{d}-\alpha_{s}$ is so small as to be experimentally uninteresting. We therefore write the transition criterion for pseudosteady flow $\left(\alpha_{\mathrm{ps}}^{*}\right)$ as

$$
\alpha_{\mathrm{ps}}^{*}=\alpha_{\mathrm{s}}\left(M_{1}, \gamma\right) \approx \alpha_{\mathrm{d}}\left(M_{1}, \gamma\right) \text {. }
$$

A common misconception is that viscous effects at the wall always provide a path of information between $T$ and $P$. It is made plain in a later section that this is not so.

The weak-reflection region of overlap $\left(M_{1}<2\right)$ poses a problem in this connection. Here, a Mach reflection is associated with a subsonic region 3. Thus, the information condition does not provide an argument against the persistence of a Mach reflection once it has been set up near the tip. An experiment that might test this possibility is one in which the tip of the wedge is made a little sharper (wedge concave near the tip). Henderson \& Lozzi (1979) performed such experiments, but these were only in the strongreflection region, where this effect is not to be expected.

\section{Steady Flow}

THE AREA OF OVERLAP WITH SUPERSONIC REGION $3\left(M_{1}>2.4\right)$ Figure 5 shows a Mach reflection in this range. The expansion wave from the trailing edge of the shock-generating wedge is refracted by the reflected shock $R$ and strikes 
the vortex sheet $V$. If it were not for this expansion, the vortex sheet would asymptotically approach a line parallel to the wall. The expansion causes $V$ to curve more strongly away from the wall, thus forming a sonic throat with the wall. Hence, an information path exists from the trailing edge along the leading characteristic to the subsonic region upstream of the sonic point and thus to P. Information about the size of the wedge can be communicated to $P$. The information condition therefore does not provide an impediment to Mach reflection in steady flow, and we thus expect the transition criterion for strong reflection in steady flow $\left(\alpha_{\mathrm{st}}^{*}\right)$ to be

$$
\alpha_{\mathrm{st}}^{*}=\alpha_{\mathrm{N}}\left(M_{1}, \gamma\right) \text {. }
$$

This has been amply confirmed by experiment (Henderson \& Lozzi 1975, 1979, Hornung \& Kychakoff 1977, Hornung et al. 1979, Hornung \& Robinson 1982). The experimental points from some of these studies are shown in Figure 16 for comparison with Equation (18).

The distinction between $\alpha_{\mathrm{ps}}^{*}$ and $\alpha_{\mathrm{st}}^{*}$ is ignored by most textbooks on gasdynamics, though it was already suspected by von Neumann (1943) and discussed at some length by Courant \& Friedrichs (1948).

THE AREA OF OVERLAP WITH $1.48<M_{1}<2.4$ Between $M_{1}=2$ and $M_{1}=2.4$, the difference between $\alpha_{N}$ and $\alpha_{d}$ is so small as to be experimentally unresolvable. In the range $1.48<M_{1}<2$, three reflection types are possible. Two of these are regular, one with weak and one with strong reflected shock, and the third is a Mach reflection [see the $(\theta, p)$-plane in Figure 15]. The Mach reflection has a subsonic region 3, and the regular reflection with weak shock has a supersonic region 3 . In order to force the Mach reflection, it is therefore necessary to constrain the flow downstream, which thus raises the back pressure (see Henderson \& Lozzi 1979). In unimpeded flow the regular reflection persists up to $\alpha=\alpha_{\mathrm{d}}$.

\section{DOUBLE MACH REFLECTION}

Double Mach reflection was first observed by Smith (1945) and White (1951). It was further studied in some detail by Gvozdeva et al. (1969), who related it to real-gas effects. While such effects can be responsible for double Mach reflection, its occurrence does not depend on them.

\section{The Reason for the Occurrence of Double Mach Reflection}

Consider a Mach reflection in the strong shock range $M_{1}>2.4$ in pseudosteady flow. In the frame of reference of $P$, region 3 is supersonic and the wall has a finite component of velocity normal to its own plane (see Figure 17). Let us assume for the moment that the flow in region 4 is 
Figure 17 Pseudosteady Mach reflection. For $(\theta, p)$-plane, see Figure 8.

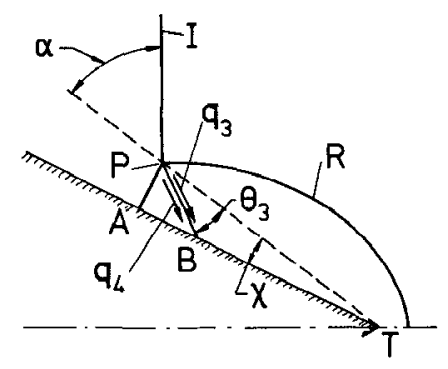

uniform, so that $\mathrm{PB}$ is a straight vortex sheet that makes an angle $\theta_{3}$ with the triple-point path. This angle is determined in terms of $M_{1}, \gamma$, and $\alpha$ by the shock-locus intersection:

$$
\theta_{3}=\theta_{3}\left(M_{1}, \gamma, \alpha\right) \text {. }
$$

Thus, the position of the point $B$ and therefore its velocity $q_{B}$ are known in P's reference frame:

$$
\mathbf{q}_{\mathrm{B}}=\mathbf{q}_{\mathrm{B}}\left(M_{1}, \gamma, \alpha, \chi\right) \text {. }
$$

The assumption that the flow in region 4 is uniform and the wall impermeability condition mean that the velocity component of the wall normal to its own plane, $q_{\mathrm{w}}$, is equal to the component of $\mathbf{q}_{\mathrm{B}}=\mathbf{q}_{4}$ normal to the wall:

$$
q_{\mathrm{w}}=q_{\mathrm{B}} \sin \left(\theta_{3}+\chi\right) \text {. }
$$

However, the velocity along the vortex sheet in region $3\left(q_{3}\right)$ is different from that in region $4\left(q_{B}\right)$. Therefore, the component of $q_{3}$ normal to the wall is not the same as the corresponding component of the wall's velocity. This inevitably leads to the need for the flow along PB in region 3 to be accelerated away from or toward the wall, depending on whether $q_{3}-q_{4}$ is positive or negative. In the frame of reference of $B$, this boils down to the need for a deflection of the flow in the vicinity of $B$ to a direction parallel to the wall.

In the strong-reflection range, we have $q_{3}-q_{4}>0$. The flow in region 3 therefore has to be decelerated and deflected (mostly) toward the wedge tip. If the relative speed $q_{3}-q_{4}$ is smaller than the speed of sound $a_{3}$, the deflection takes place without a shock being generated. The resulting configuration is either single Mach reflection or what is sometimes called complex Mach reflection. If, on the other hand $q_{3}-q_{4}>a_{3}$, the deflection occurs via a shock, and we speak of double Mach reflection. The shock may 


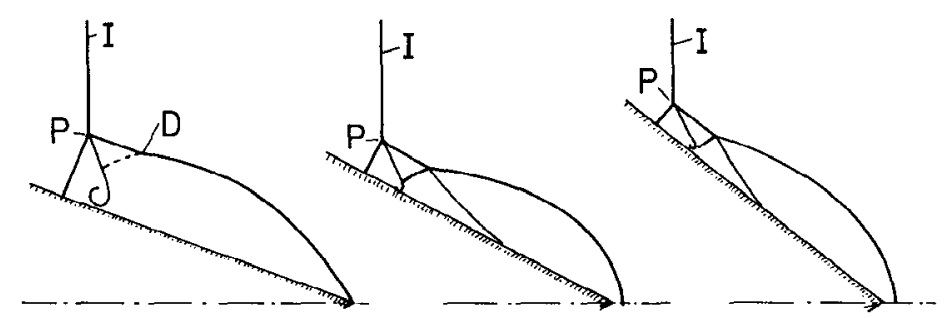

Figure 18 Complex (left) and double Mach reflections. The second Mach stem may be curved (center) or straight (right).

either be straight or curved, depending on the shock strength required for the deflection (see Figure 18).

Complex Mach reflection is an interesting case, because while region 3 is supersonic in P's reference frame, it is subsonic in B's reference frame. Hence, the reflected shock is straight up to the point $D$ at which the signal from B has caught up to it (Figure 18).

The requirement that the flow pattern be pseudosteady, together with the assumption that the whole of region 4 is uniform, amounts to the assumption that the Mach stem is straight and normal to the wall. That this is so may be seen by considering the sink points for the various regions of this reflection in pseudosteady space (see Figure 19). The assumption that $S$ is normal to the wall at the triple point is made by Law \& Glass (1971) in order to calculate the angle $\chi$. Glass and his collaborators have built

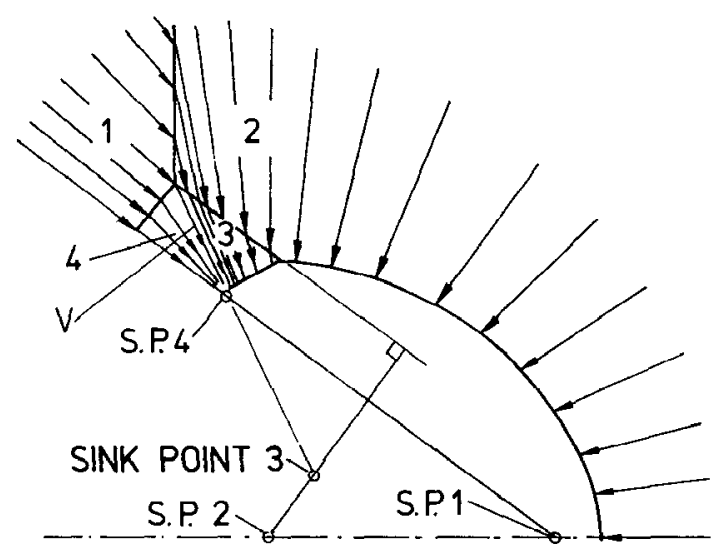

Figure 19 Double Mach reflection in pseudosteady similarity space with the sink points of the uniform-flow regions indicated. 


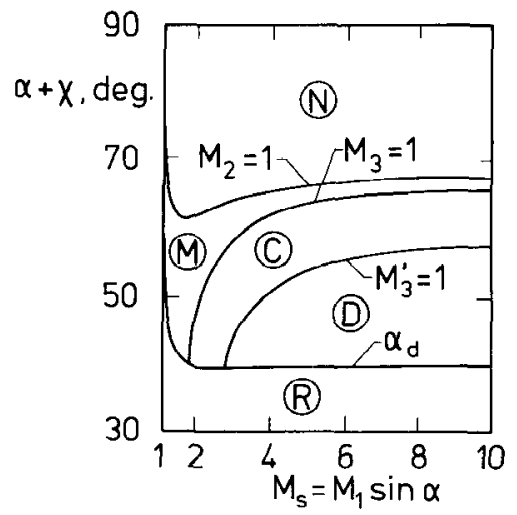

Figure 20 The structure of the direct Mach-reflection range (cf. Figure 16) for pseudosteady flow at $\gamma=1.4$. (After BenDor 1978.) $R=$ regular, $\mathrm{D}=$ double Mach, $\mathrm{C}=$ complex Mach, $\mathbf{M}=$ single Mach, and $\mathrm{N}=$ no reflection. $M_{3}^{\prime}$ is the Mach number relative to the second triple point.

up a considerable body of knowledge on these more complicated Mach reflections. In particular, the domains in which double and complex Mach reflection are to be expected on the basis of the Law/Glass assumption have been mapped out in detail by Ben-Dor (1978). This is shown in Figure 20 in the form of a plot of the angle between the incident shock and the wedge $(\alpha+\chi)$ against the incident-shock Mach number $\left(M_{\mathrm{s}}=M_{1} \sin \alpha\right)$. The boundary between double and complex Mach reflection in this diagram is calculated by Ben-Dor from the condition that the flow in region 3 is sonic in the frame of reference of the second triple point (see also Ben-Dor \& Glass 1979, 1980, Ando 1981). This condition is slightly different from the view taken here, that region 3 should be sonic in the reference frame of $B$ at transition.

It should be pointed out that all the computations of the domains of the various reflection configurations and their boundaries (except for the boundary indicating failure of regular reflection) rely directly or indirectly on assumptions of how the wall impermeability condition influences the angles of the triple-point paths. The above description of the reasons for the occurrence of double Mach reflection makes it clear that such assumptions lead only to relatively crude approximations. This is further illustrated in recent work by Shirouzu \& Glass (1984), who attempted a refinement of the assumptions by taking into account the curvature of the Mach stem.

\section{The Curling Up of the Vortex Sheet}

The need for the flow from region 3 to be deflected at $B$ means that the pressure is higher at $B$ than to the left of $B$ (see Figure 21). In the frame of reference of $B$, the gas to the left of $B$ (region 4 , considered uniform) is at rest. The pressure distribution corresponding to this flow is as sketched in Figure 21. Such a pressure distribution causes the flow to be accelerated 

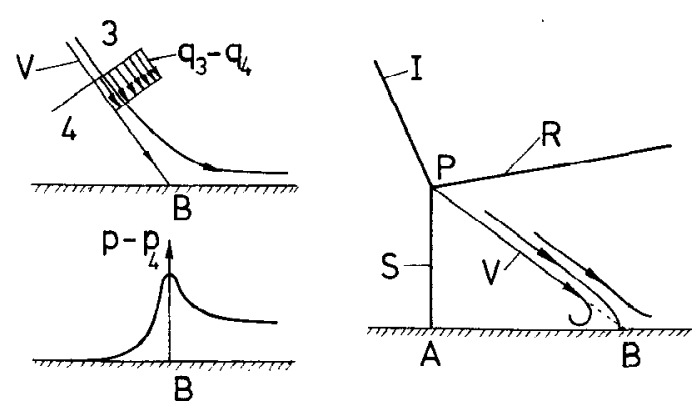

Figure 21 Pseudosteady Mach reflection viewed from B's reference frame. The pressure gradient to the left of $\mathbf{B}$ causes the vortex sheet to roll up.

away from $B$ in all directions (stagnation point), with the result that the vortex sheet is deflected to the left and the dividing streamline, which is then free of vorticity, strikes the wall at right angles. Occasionally, the pressure gradient to the left of B can be so severe as to drive a wall jet to the left so strongly that it influences the Mach stem (see, for example, Sandeman et al. 1980, Collela \& Glaz 1984). This phenomenon (stem bulge) may be expected to be impeded by viscous effects.

\section{Computation of the Flow Field}

A tempting approach to the solution of pseudosteady flows is the numerical solution of Equations (11). Computations of this kind have been presented, for example, by Schneyer (1975), Kutler \& Shankar (1977), who also present a review of the subject, Shankar et al. (1977), and (more recently) Collela \& Glaz (1984). The results of the first three of these were compared with experiments by Ben-Dor \& Glass (1978). While this comparison shows general agreement, it also reveals discrepancies that may be due to viscous, real-gas, or numerical viscosity effects. The more recent work of Collela \& Glaz (1984) shows that the numerical approach predicts an amazing degree of complexity and detail, including multiple Mach reflection with stem bulge and vorticity concentrations. Publications on this subject are in press from this group and should be of interest to all students of Mach reflection.

An important field where computation of the flow field is necessary is that of weak reflection. This is because the downstream flow often contains subsonic regions, so that the downstream boundary conditions matter. An analytical technique using the method of strained coordinates was developed for very weak shocks by Obermeier (1984). The results of a particular computation were compared with the experiments of Henderson \& Siegenthaler (1980) and showed promising agreement. 


\section{EFFECTS DUE TO THE WALL BOUNDARY CONDITIONS}

\section{The Effect of Viscosity and Heat Transfer in "Pseudosteady" Flow}

The introduction of any length scale independent of $d$ into a pseudosteady flow must break the pseudostationarity, i.e. the self-similarity. With viscosity, such an independent length, $l_{\mathrm{v}}=\mu /(\rho q)$, is introduced. Hence the quotes in the title of this section.

The experimental results on pseudosteady flow indicate that the transition from regular to Mach reflection occurs at values of $\alpha$ that lie significantly above $\alpha_{d}$ when the reflection is arranged to occur off a solid surface (see, for example, Bleakney \& Taub 1949, Henderson \& Lozzi 1975, Takayama \& Sekiguchi 1977). However, when the reflection occurs (as, for example, in the case of symmetrically interacting shocks) off a symmetry plane (see Figure 22), such as in the experiments of Smith (1959) and Henderson \& Lozzi (1975), the no-slip condition at the reflection surface and associated viscous effects are avoided. In these cases the transition was found to occur - to within experimental accuracy - at the theoretical value of $\alpha_{d}$. The persistence of regular reflection to beyond $\alpha_{d}$ is therefore a wall effect.

Consider a regular pseudosteady reflection in the frame of reference of $\mathbf{P}$ (see Figure 23). There exists no shear along section EP of the wall, but since $q_{3}<q_{1}$, a velocity discontinuity exists in an inviscid flow across the surface along PF. The no-slip condition and shear viscosity therefore cause a boundary layer to develop from $P$, in which the flow depends on all five of the parameters in Equation (1). This boundary layer is of the type in which the wall velocity is greater than the free-stream velocity (in the reference
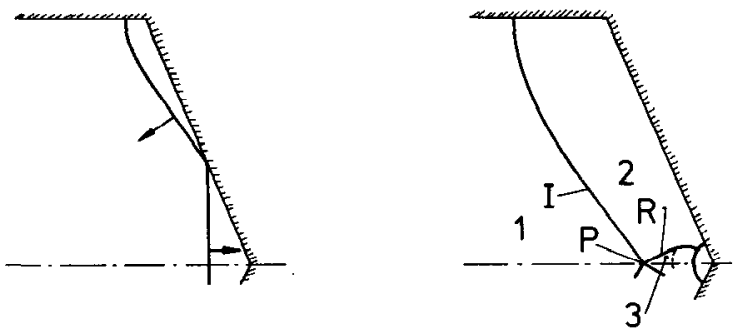

Figure 22 Arrangement for setting up pseudosteady reflection off a symmetry plane to avoid viscous effects. (left) Early. (right) Later. 
frame in which the flow is steady), so that its displacement thickness is negative. Since the wall temperature is also usually lower than the recovery temperature [the free stream (region 3) having been shock heated from the temperature of region 1, i.e. the wall temperature], the magnitude of the negative displacement thickness is increased by heat transfer to the wall.

The influence that this effect will have on the transition angle may be estimated by the following argument. The effective shape of the wall to the right of $\mathrm{P}$ as seen by the far field (Figure 23) is such as to require the flow deflection through the reflected shock to be smaller than that through the incident shock by a small angle $\varepsilon$ (see Hornung et al. 1979). Hence, the possibility of a regular reflection ceases to exist not at $\alpha_{d}$, but at a condition where the maximum-deflection angle across the reflected shock is $\theta_{2}-\varepsilon$ (Figure 23). This causes the transition angle to be raised by viscosity to a value

$$
\alpha_{\mathbf{v}}^{*}>\alpha_{\mathbf{p s}}^{*}=\alpha_{\mathrm{s}}
$$

The subscripts are intended to distinguish viscous from pseudosteady (and therefore inviscid) values.

It is sometimes argued that the presence of a boundary layer on the wall provides a path for the information from the wedge tip to reach the reflection point. In fact, this is not so, because the flow speed (and the Mach number) in P's reference frame, against which the signal has to travel, is even higher in the boundary layer than in the free stream.

Provided that the boundary layer is laminar, its displacement thickness
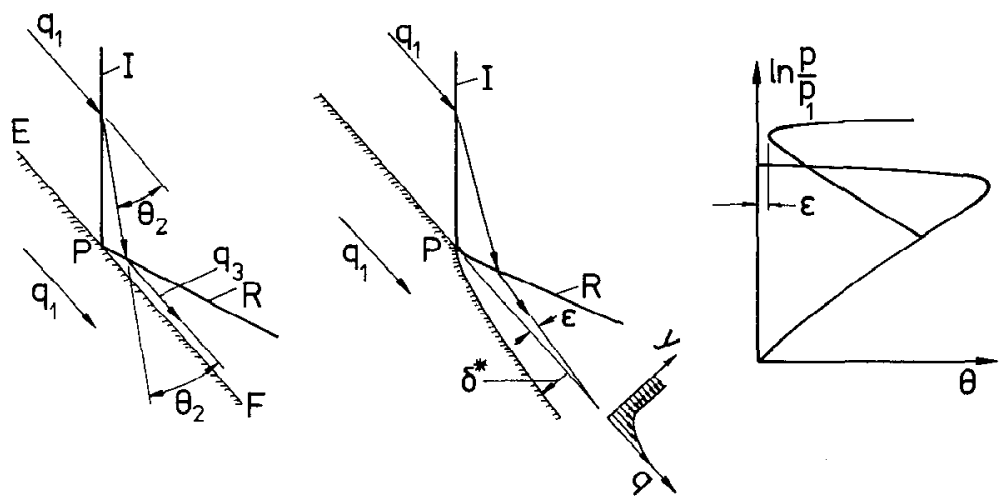

Figure 23 Regular reflection viewed from P's reference frame. (left) Inviscid. (center) Viscous displacement effect as seen by flow outside the boundary layer. (right) Viscosity causes transition to Mach reflection to be delayed. 


\section{HORNUNG}

$\delta^{*}$ may be shown to behave (see Becker 1961) as

$$
\delta^{*} / x=-f\left(\operatorname{Pr}, \gamma, M, T_{\mathrm{w}} / T_{0}\right)\left(l_{\mathrm{v}} / x\right)^{1 / 2},
$$

where $x$ is the distance along the wall from the point at which the boundary layer starts (in this case $\mathbf{P}$ ), and the parameters on the right-hand side are to be evaluated outside the boundary layer. If the wall temperature is equal to the static temperature in region 1 , and $\gamma$ and $M_{1}$ are held fixed, then $M_{3}, T_{03}$, $l_{\mathrm{v} 3}$ and $\mathrm{Pr}_{3}$ will also be constant for approximately constant $\alpha$. Under such conditions, it may be shown that

$$
\alpha_{\mathrm{v}}^{*}=\alpha_{\mathrm{ps}}^{*}+C\left(l_{\mathrm{v} 3} / l\right)^{1 / 2},
$$

where $C$ is a constant. The difficulty with this form is that there seems to be no local characteristic length to choose for $l$. Hornung \& Taylor (1982) chose $l$ to be the smallest resolvable length of the observer, on the basis that this is the Mach-stem length at observed transition. Their experiments confirmed Equation (24) and showed that, if extrapolated to infinite Reynolds number $\left(l / l_{\mathrm{v} 3}\right)$, it gave excellent agreement with $\alpha_{\mathrm{ps}}^{*}=\alpha_{\mathrm{d}}$, though the maximum difference between $\alpha_{\mathrm{v}}^{*}$ and $\alpha_{\mathrm{ps}}^{*}$ was more than $6^{\circ}$. This result is independent of their choice of $l$.

In fact, the problem is not quite so simple. To understand it better, consider the question of how the length of the Mach stem is modified by the presence of viscous effects at the wall. Let the inviscid (pseudosteady) value of the Mach-stem length be $L_{\mathrm{ps}}$ and its viscous value $L_{\mathrm{v}}$. For a laminar boundary layer it may be shown (see Hornung 1985) that

$$
L_{\mathrm{v}} / L_{\mathrm{ps}}=1-2 f_{4}(\tan \beta / \tan \chi)^{1 / 2}\left(l_{\mathrm{v}} / d\right)^{1 / 2},
$$

where $f_{4}$ is the function in Equation (23) evaluated in region 4, $\beta$ is the angle between $\mathrm{V}$ and the Mach stem, and $\chi$ is the angle of the triple-point path for inviscid flow.

The resulting path of the triple point of a single Mach reflection is shown in Figure 24. Whether a reflection is interpreted as a regular or a Mach reflection appears to depend on whether it is observed at a distance from the wedge tip that is smaller or greater than

$$
d_{0}=l_{\mathrm{v} 4}\left(4 f_{4}^{2} \tan \beta / \tan \chi\right),
$$

i.e. the value of $d$ at which $L_{v}=0$. However, the theory leading to Equation (25) is an asymptotic theory for high Reynolds number and assumes that the second term on the right of (25) is small compared with unity. The step from (25) to (26) is therefore incorrect because it uses the theory outside its range of validity. It is clear from (25), however, that the length to be used in Equation (24) is the distance $d_{1}$ from the tip at which observations are made. 
Figure 24 Sketch illustrating the effect of viscosity on the triple-point path. - - , inviscid flow; ___ viscous flow; -...-., according to observer assuming inviscid flow; -- --- , by the procedure of Henderson \& Gray (1981).

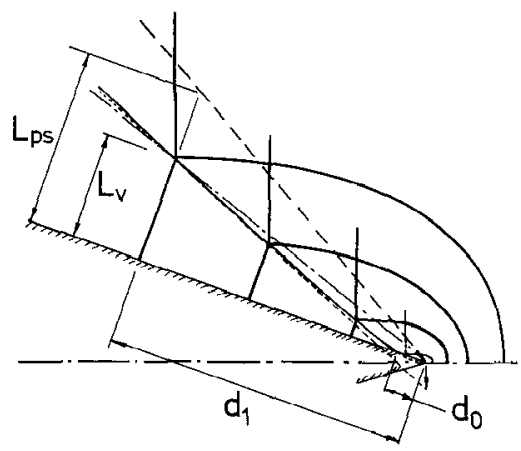

Very careful experiments made by Henderson \& Gray (1981) confirmed the behavior of the triple-point path sketched in Figure 24, inasmuch as the path's apparent origin was observed to lie below the wedge tip if extrapolated back along a straight path chosen in such a manner as to satisfy the shock-jump conditions at the observed geometry. Their interpretation of this effect was that it might be due to leakage near the tip of the wedge, whose symmetry was approximated in their experiments by contact of the sharp leading edge with a flat plate along the symmetry plane to the right.

From our discussion of the effect of shear viscosity, it may be recognized that the mechanism by which heat conduction affects shock reflection is via its effect on the displacement thickness of the boundary layer. Thus, a colder wall would act like a higher viscosity.

\section{The Effects of Surface Roughness and Porosity}

If the wall is rough, a mechanism by which momentum may be imparted to the gas near the wall is possible even without shear viscosity. Thus, roughness may cause a negative displacement thickness, and regular reflection may again be expected to persist to higher values of $\alpha$ than $\alpha_{d}$. This was shown to be indeed the case by Takayama et al. (1981), who performed experiments with different kinds of roughness. They also constructed an empirical model involving the total pressure loss due to the roughness. Excesses of the transition angle of up to $20^{\circ}$ beyond $\alpha_{d}$ could be obtained.

An effect that is closely related to that of viscosity may be obtained by letting the shock reflect off a porous wall. Since the shock system raises the pressure, the passage of the shock switches on a flow through the wall. The result is that gas leaks out of region 4 , which has the same effect as a growing negative displacement thickness. 
A theory describing this effect was constructed by Clarke (1984a) in which the porosity of the wall is modeled by Darcy's law and a second, solid wall backs up the porous material. The strength of the reflected shock is considerably attenuated compared with solid-wall behavior, and the adjustment to final equilibrium occurs in an essentially shock-free manner. The theory gives solutions for the flow field and has been shown to agree very well with experiments designed to test it (see Clarke 1984b). Clarke did not examine the transition to Mach reflection, though a computation of the effect on the transition angle appears to be within the scope of the theory.

\section{INVISCID UNSTEADY EFFECTS}

The introduction of any independent length scale breaks the pseudostationarity. Therefore, the wall effects of the previous section all cause the flow to become truly unsteady, so that the point $P$ is accelerated. A frame of reference attached to $P$ would therefore not be an inertial frame, and body forces would need to be introduced to correct for this. While this is only of minor importance in the weak unsteadinesses considered so far, it becomes completely impossible to use the pseudosteady flow ideas for truly unsteady flows such as are encountered, for example, when a plane shock strikes a circular cylinder transversely, or when a spherical shock strikes a plane surface. It must therefore not be expected (as is sometimes done) that pseudosteady- or steady-flow criteria have any meaning at all in unsteady situations.

Short of computing the whole flow field in a space of at least three independent variables, the only theoretical model available for predicting the behavior of such flows is that of Whitham (1957) for the propagation and interaction of curved shocks. Heilig (1969) performed very detailed experiments on the interaction of a plane shock with a circular cylinder. At and after first contact, regular reflection is observed, and at a particular angle $\alpha_{1}$ between the shock and the cylinder surface a Mach stem begins to grow. Heilig found that this value of $\alpha_{1}$ exceeds $\alpha_{d}$ by an amount that varies with $M_{\mathrm{s}}$ and reaches a maximum of $13^{\circ}$ at $M_{\mathrm{s}}=1.2$. He also computed the triple-point path using Whitham's theory and found good agreement for the case of strong shocks.

A number of further experimental investigations of unsteady shock reflection have been made on both convex and concave surfaces. Only a few of these are mentioned for further reference: Henderson \& Lozzi (1979), Itoh et al. (1981), Ben-Dor et al. (1980), Takayama et al. (1981). On concave surfaces it is possible for the reverse transition from Mach to regular reflection to occur. Also, the angle at which this occurs is substantially smaller than $\alpha_{d}$. Here, as in other experiments, information enabling the 
determination of the viscous and heat-transfer parameters necessary for correct interpretation is often omitted.

\section{REAL-GAS EFFECTS}

\section{Rarefied Gases}

At this point we depart from our restriction to continua in order to describe an interesting effect due to gas rarefaction observed by Walenta (1983). Estimates of the effect of shock thickness on the flow in the very near vicinity of the triple point had been made by Sternberg (1959), who concluded that it was negligible in the flow regime he considered. Walenta's experiments (see also Schmidt 1985) investigate the interaction of a plane shock with a wedge in a range where the typical values of $d$ are around 100 mean free paths. He shows that the shock angles at the triple point differ markedly from those expected from inviscid continuum theory and that, furthermore, the constancy of $\theta$ and $p$ across the vortex "sheet" does not apply any more. This is not surprising, because the vortex sheet is now not a thin shear layer, but one that grows rapidly due to viscous action in this situation. [This latter effect has been disregarded so far because its effect on transition in continuum flows is minor (see also Sternberg 1959, Sakurai 1964).]

The most interesting aspect of Walenta's results is that the triple-point path approximately follows a straight line that does not pass through the wedge tip but intersects the wedge surface at a distance from the tip of some 50 to 100 mean free paths. He explains this as follows (private communication): The reflected shock starts at the tip as a cylindrical shock. The speed of a curved shock whose radius of curvature $R$ is not very much larger than its thickness $\delta$ is proportional to $1-\delta / R$. It therefore lags behind the incident shock until $R$ becomes large, even in a situation that should give Mach reflection according to continuum theory. In these experiments too, the boundary conditions at the wall as regards slip and heat transfer could play a significant part (see Hornung 1985).

\section{High-Temperature Continuum Effects}

If the shock speed is so high that the gas is vibrationally excited, dissociated, or ionized, we distinguish between two cases. In the first of these, thermodynamic equilibrium is assumed to be maintained outside of very thin shocks. Since the equilibrium condition is changed from that of a perfect gas, the transition angles will be modified also. No new length scale is introduced into the flow, however, so that the new transition angles may be computed for steady and pseudosteady flows just as before. This has been done, for example, by Gvozdeva et al. (1969), Hornung et al. (1979), 
and Ben-Dor \& Glass (1980). Where satisfactory agreement with experiment was not observed, the direction of the discrepancy may be explained by viscous effects. Both $\alpha_{d}$ and $\alpha_{N}$ are increased by endothermic real-gas effects. (The opposite is true for exothermic effects, such as in detonation waves.)

The second effect is that due to relaxation. If the relaxation time is resolvable by the observer, then a new, independent length (the relaxation length) is introduced into the problem. This was shown by Sandeman et al. (1980) to have a considerable effect in dissociating nitrogen and in ionizing argon when the relaxation length is comparable with the distance $d$.

\section{CONCLUSIONS}

The last decade has seen extensive developments in the study of shock reflection. These have led to a satisfactory understanding of the failure of regular reflection in the strong-reflection range for inviscid steady and pseudosteady flow. However, there remain a number of unanswered questions even in steady and pseudosteady flow. This is especially true for weak shock reflection, where the effect of the downstream boundary conditions needs to be examined in more depth. A further uncertainty lies in the assumptions usually made to predict the triple-point paths and the resulting transitions to complex and double Mach reflection. Some of these difficulties may be associated with the faulty interpretation of experimental results that often occurs through ignoring the effects of shear viscosity.

In this review several effects have been singled out that cause the pseudostationarity of shock reflection to be broken by introducing an independent length scale. The most important of these are the effects due to shear viscosity and heat conduction at the wall because they strongly affect the interpretation of nominally pseudosteady results. A solution for the effect of viscosity on the triple-point path, given in this review, illustrates how such a misinterpretation may occur. Future experiments must take this into account.

\section{ACKNOWLEDGMENTS}

I wish to thank Wladek Fiszdon and Reinhard Niehuis for the care with which they read and corrected the manuscript.

\section{Literature Cited}

Ando, S. 1981. Domains and boundaries of pseudosteady oblique shock reflections in carbon dioxide. UTIAS Tech-Note No. 231 , Inst. Aerosp. Stud., Univ. Toronto Becker, E. 1961. Instationäre Grenzschichten hinter Verdichtungsstößen und Expansionswellen. Prog. Aeronaut. Sci. 1 : 104-73

Ben-Dor, G. 1978. Regions and transitions of nonstationary oblique shock-wave diffractions in imperfect and perfect gases. 
UTIAS Rep. No. 232, Inst. Aerosp. Stud., Univ. Toronto

Ben-Dor, G., Glass, I. I. 1978. Nonstationary oblique shock-wave reflections: Actual isopycnics and numerical experiments. AIAA J. 16:1146-53

Ben-Dor, G., Glass, I. I. 1979. Domains and boundaries of nonstationary oblique shock-wave reflexions: 1 . Diatomic gas. $J$. Fluid Mech. 92:459-96

Ben-Dor, G., Glass, I. I. 1980. Domains and boundaries of non-stationary oblique shock-wave reflexions : 2 . Monatomic gas. J. Fluid Mech. 96:735-56

Ben-Dor, G., Takayama, K., Kawauchi, T. 1980. The transition from regular to Mach reflexion and from Mach to regular reflexion in truly non-stationary flow. $J$. Fluid Mech. 100:147-60

Bleakney, W., Taub, A. H. 1949. Interaction of shock waves. Rev. Mod. Phys. 21:584605

Bryson, A. E., Gross, R. F. W. 1961. Diffraction of strong shocks by cones, cylinders and spheres. J. Fluid Mech. 10:1-16

Clarke, J. F. 1984a. Regular reflection of a weak shock wave from a rigid porous wall. Q. J. Mech. Appl. Math. 37:87-111

Clarke, J. F. 1984b. The reflection of weak shocks from absorbent surfaces. Proc. $R$. Soc. London Ser. A 396:365-82

Collela, P., Glaz, H. M. 1984. Numerical calculation of complex shock reflections in gases. Presented at Mach Reflection Symp., 4th, Sendai, Jpn.

Courant, R., Friedrichs, K. O. 1948. Supersonic Flow and Shock Waves. New York: Interscience. $464 \mathrm{pp}$.

Griffith, W. C. 1981 . Shock waves. J. Fluid Mech. 106:81-101

Grozdeva, L. G., Bazhenova, T. V., Predvoditeleva, O. A., Fokeev, V. P. 1969. Mach reflection of shock waves in real gases. Astron. Acta 14: 503-8

Heilig, W. 1969. Theoretische und experimentelle Untersuchungen zur Beugung von Stosswellen an Kugeln und Zylindern. Dissertation. Univ. Karlsruhe. 114 pp. See also 1977. A result concerning the transition from regular reflection to Mach reflection of strong shock waves. In Shock Tube and Shock Wave Research, ed. B. Ahlborn, A. Herzberg, D. Russell, pp. 288-96. Seattle: Univ. Wash. Press

Henderson, L. F., Gray, P. M. 1981. Experiments on the diffraction of strong blast waves. Proc. R. Soc. London Ser. A 377:363-78

Henderson, L. F., Lozzi, A. 1975. Experiments on transition to Mach reflexion. J. Fluid Mech. 68:139-55

Henderson, L. F., Lozzi, A. 1979. Further experiments on transition to Mach reflexion. J. Fluid Mech. 94:541-59
Henderson, L. F., Siegenthaler, A. 1980. Experiments on the diffraction of weak blast waves. The von Neumann paradox. Proc. R. Soc. London Ser. A 369:537-55

Hornung, H. G. 1985. The effect of viscosity on the Mach stem length in unsteady shock reflection. In Unsteady Fluid Motion, Lecture Notes in Physics. In press

Hornung, H. G., Oertel, H., Sandeman, R. J. 1979. Transition to Mach reflexion of shock waves in steady and pseudosteady flow with and without relaxation. $J$. Fluid Mech. 90:541-60

Hornung, H. G., Robinson, M. L. 1982. Transition from regular to Mach reflection of shock waves. Part 2. The steady-flow criterion. J. Fluid Mech. 123:155-64

Hornung, H. G., Taylor, J. R. 1982. Transition from regular to $M$ ach reflection of shock waves. Part 1 . The effect of viscosity in the pseudosteady case. J. Fluid Mech. 123: 143-53

Hornung, H. G., Kychakoff, G. 1977. Transition from regular to Mach reflexion of shock waves in relaxing gases. In Shock Tube and Shock Wave Research, ed. B. Ahlborn, A. Herzberg, D. Russell, pp. 297302. Seattle: Univ. Wash. Press

Itoh, S., Okazaki, N., Itaya, M. 1981. On the transition between regular and Mach reflection in truly non-stationary flows. $J$. Fluid Mech. 108:383-400

Jones, D. M., Martin, P. M. E., Thornhill, C. K. 1951. A note on the pseudo-stationary flow behind a strong shock diffracted or reflected at a corner. Proc. R. Soc. London Ser. A 209: 238-48

Kawamura, R., Saito, H. 1956. Reflection of shock waves -1 . Pseudostationary case. $J$. Phys. Soc. Jpn. 11:584-92

Kutler, P., Shankar, V. 1977. Diffraction of a shock wave by a compression corner. Part I : Regular reflection. AIAA J. 5:197-202

Law, C. K., Glass, I. I. 1971. Diffraction of strong shock waves by a sharp compressive corner. CASI Trans. 4:2-12

Lighthill, M. J. 1949. On the diffraction of blast I. Proc. R. Soc. London Ser. A 198: 454-70

Mach, E. 1878. Ober den Verlauf von Funkenwellen in der Ebene und im Raume. Sitzungsber. Akad. Wiss. Wien 78:819-38

Obermeier, F. 1984. Development and propagation of weak Mach-shock waves. Presented at Mach Reflection Symp., 4th, Sendai, Jpn.

Pack, D. C. 1964. The reflexion and diffraction of shock waves. J. Fluid Mech. 18: 549-76

Sakurai, A. 1964. On the problem of weak Mach reflection. J. Phys. Soc. Jpn. 19: 1440-50

Sandeman, R. J., Leitch, A., Hornung, H. 
1980. The influence of relaxation on transition to Mach reflection in pseudosteady flow. In Shock Tubes and Waves, ed. A. Lifshitz, J. Rom, pp. 298-307. Jerusalem: Magnes

Schmidt, B. 1985. Stosswellenübergang von regulärer Reflektion zur Mach Reflektion auf der Flanke eines Keils. Z. Angew. Math. Mech. 65:234-36

Schneyer, G. P. 1975. Numerical simulation of regular and Mach reflection. Phys. Fluids 18:1119-24

Shankar, V. S., Kutler, P., Anderson, D. A. 1977. Diffraction of shock waves by a compression corner, Part II-Single Mach reflection. AIAA Pap. No. 77-78

Shirouzu, M., Glass, I. I. 1984. An assessment of recent results on pseudo-stationary oblique-shock-wave reflections. Presented at Mach Reflection Symp., 4th, Sendai, Jpn.

Smith, L. G. 1945. Photographic investigation of the reflection of plane shocks in air. OSRD Rep. No. 6271, Off. Sci. Res. Dev., Washington, DC

Smith, W. R. 1959. Mutual reflection of two shock waves of arbitrary strengths. Phys. Fluids 2: 533-41

Sternberg, J. 1959. Triple-shock-wave inter- sections. Phys. Fluids 2:179-206

Takayama, K., Sekiguchi, H. 1977. An experiment on shock diffraction by cones. Rep. Inst. High Speed Mech., Tohoku Univ. 336:53-74

Takayama, K,, Gotoh, J., Ben-Dor, G. 1981. Influence of surface roughness on the shock transition in quasistationary and truly non-stationary flows. In Shock Tubes and Waves, ed. C. F. Treanor, J. G. Hall, pp. 326-34. Albany: State Univ. N.Y. Press

von Neumann, J. 1943. Oblique reflection of shocks. Explos. Res. Rep. No. 12, Dep. Navy, Washington, DC. See also Collected Works, 6:238-99. Oxford: Pergamon (1963)

Walenta, Z. A. 1983. Formation of the Machtype reflection of shock waves. Arch. Mech. $35: 187-96$

White, D. R. 1951. An experimental survey of the Mach reflection of shock waves. Princeton Univ. Dept. Phys. Tech. Rep. II10, Princeton, N.J.

Whitham, G. B. 1957. A new approach to problems of shock dynamics. Part 1. Twodimensional problems. J. Fluid Mech. 2: 145-71 
Annual Review of Fluid Mechanics
Volume 18,1986

\section{CONTENTS}

KeIth SteWARTSON : His LIFE AND Work, J. T. Stuart 1

Strange Atrractors in Fluids: ANOTHER VIEW, John Guckenheimer 15

Regular and Mach Reflection of Shock Waves, Hans Hornung 33

Gravity CurRents in Rotating Systems, $R$. $W$. Griffiths

Eddies, Waves, Circulation, AND Mixing: Statistical Geofluid Mechanics, Greg Holloway 91

Wind-Wave Prediction, Rodney J. Sobey 149

THREE-Dimensional AND UnSTEADY Boundary-LAYER Computations, J. Cousteix 173

STEady and UnSTEady Boundary-Layer SeParation, $F$. T. Smith 197

VorteX Flows in AERodynamics, J. H. B. Smith 221

Flows Far From Equilibrium via Molecular Dynamics, Denis $J$. Evans and William G. Hoover

The Continental-Shelf Bottom Boundary Layer, William $D$. Grant and Oie S. Madsen

INTERACTION OF Flows With THE CRYSTAL-Melt INTERFACE, M. E. Glicksman, S. R. Coriell, and G. B. McFadden

Characteristic-Based Schemes for the Euler Equations, P. L. Roe

Marine Propellers, Justin E. Kerwin 367

Critical Layers in Shear Flows, $\boldsymbol{S}$. A. Maslowe $\mathbf{4 0 5}$

Vorticity Dynamics of the Oceanic General. Circulation, Peter B. Rhines

INDEXES

Subject Index

Cumulative Index of Contributing Authors, Volumes 1-18 507

Cumulative Index of Chapter Titles, Volumes 1-18 